\title{
ONLINEKURS „INTERKULTURELLES LERNEN UND ARBEITEN“ ALS EIN BAUSTEIN DER INTERKULTURELLEN QUALIFIZIERUNG - ANMERKUNGEN ZUM EINSATZ
}

\author{
Olga Rösch, Margit Scholl, Bettina Strewe, Christian Niemczik, Anja Teske
}

\section{Zusammenfassung}

Im Rahmen des Verbundprojekts „InterKomp KMU 2.0“ ist im Teilprojekt „Technik und Kultur“ der TH Wildau $[\mathrm{FH}]^{1}$ ein computergestütztes Lehrwerk zu interkulturellen Kompetenzen für KMU und insbesondere für KKU in Brandenburg entstanden, das inhaltlich und methodisch sowohl mehreren Anforderungen an Weiterbildungsangebote im Themenfeld Interkulturalität entspricht als auch dem Lehr- und Lernformat Blended Learning (BL) genügt. Der modular aufgebaute Onlinekurs bildet thematisch die wichtigsten Schwerpunkte des Bereiches Interkulturelles Lernen $a b$, stellt eine bereichernde Ergänzung für jede interkulturelle Bildungsmaßnahme dar und ist für Nutzer frei und kostenlos zugänglich. Für das selbstständige Lernen ist der Onlinekurs eine gute Möglichkeit, eine größere Sensibilität für interkulturellen Kontexte zu entwickeln und das Grundlagenwissen auf dem Gebiet der interkulturellen Kommunikation anzueignen. Allerdings muss er als Lehrund Lernmaterial in den Gesamtzusammenhang der interkulturellen Bildung eingebettet und der Lernprozess durch diskursive Lernmethoden in den Präsenzphasen professionell begleitet werden, um das Lernziel interkultureller Handlungskompetenz zu erreichen.

\begin{abstract}
The sub-project "'Technology and Culture' at the UAS Wildau" forms part of the joint project "Interkomp KMU 2.0". It has generated a computerized textbook providing an online course on intercultural competences for small and medium-sized enterprises (SMEs), with a particular focus on micro and small enterprises (MSEs) in Brandenburg. The contents and methodology of this course satisfy the various demands for further education in the field of interculturalism and conform to the teaching and learning formats of Blended Learning (BL). With its modular structure, the course takes a thematic approach, mapping out most important areas for intercultural study and work. It represents a valuable addition to any intercultural training programme and is universally accessible to users free of charge. The course is a useful resource for self-directed learning, providing an opportunity to develop greater sensitivity to intercultural contexts and acquire a basic grounding in the field of intercultural communication. However, its teaching and learning materials need to be integrated into the overall context of intercultural education and the learning process should go hand in hand with discursive learning methods applied in the course of professional on-campus training periods. In this way the learning objectives of intercultural competence can be achieved.
\end{abstract}

\section{EINLEITUNG}

Die Bedeutung interkultureller Kompetenz bei der Verhandlungskommunikation, Vertragsgestaltung oder Durchführung von internationalen Projekten ist unbestritten. Interkulturelle Kompetenz ist zu einer unverzichtbaren Qualifikationskomponente in der Managerausbildung geworden und gilt inzwischen als Ausweis für Professionalität bei der Abwicklung von internationalen Geschäften. Dementsprechend ist der Bedarf an Kulturwissen bzw. Fachwissen der In- terkulturalisten in den letzten 20 Jahren stark gestiegen.

Die Hochschulen begegneten dem Bedarf der globalisierten Märkte und einer Multikulturalisierung der Gesellschaft mit Intensivierung ihrer Forschung zur interkulturellen Kommunikation ${ }^{2}$, durch neue innovative Studiengänge mit interkulturellen Schwerpunktsetzungen sowie mit studienbegleitenden Lehrangeboten in verschiedenen Formaten. Die größeren international agierenden Wirtschaftsunternehmen, aber auch nichtkommerzielle Einrichtungen, Institutionen und öffentlicher Dienst reagierten auf den Bedarf mit entsprechenden Fortbildungsmaßnahmen (vgl. z.B. Grünhage-Monetti, 2005, Otten/ Scheitza/ Cnyrim, 2007 u.a.).

Bildungsangebote mit interkulturellen Schwerpunkten umfassen thematisch ein breites Spektrum und werden methodisch in vielfältigen Formaten zwischen klassischem Unterricht und Lehre über Trainings bzw. Seminare bis zu Coachings und Beratungen realisiert. Das Lehrfach

'Der Anfang der Arbeiten an dem Themenkomplex „Technik und Kultur“ wurde an der TH Wildau 2008 durch eine gleichnamige Fachtagung gemacht, die von Prof. Dr. Olga Rösch, Leiterin des Instituts für interkulturelle Kommunikation, konzipiert und durchgeführt wurde. Die Tagungsbeiträge sind in der Wildauer Schriftenreihe Interkulturelle Kommunikation, Bd. 6 veröffentlicht, s. Rösch (2008).

Wir können heute auf eine Vielzahl von Fachpublikationen aus dem deutschsprachigen Raum zurückgreifen, die die Entwicklungen reflektieren, hier z.B. einige Veröffentlichungen der letzten 7 Jahre: Bolten 2007, Moosmüller 2007, Rothlauf 2009, Kutschker/ Schmid 2010, Barmeyer 2010, Weidemann/ Straub/ Notnagel 2010, Koch 2012, Busch 2013, Thomas 1995-2014 u.a.. 
Interkulturelle Kommunikation bzw. das Interkulturelle Training hat in der Folge für die Vermittlung von Grundlagen seinen eigenen Themenkanon und ein bestimmtes Methodenarsenal entwickelt (vgl. z.B. Roth \& Köck 2004, Thomas \& Hößler 2007, Heringer 2007, Lüsebrink 2008, Eß 2010, Müller/Gelbricht 2013 u.a.).

Das FHProfUnt ${ }^{3}$-Verbundprojekt „Konzipierung von modularen Weiterbildungsangeboten für $\mathrm{KMU}$ an der Schnittstelle von Technik und Kultur zum Thema, Internationales IT-gestütztes Projekt- und Wissensmanagement im multikulturellen Umfeld' (InterKomp KMU 2.0)“ wurde von Juli 2010 bis Oktober 2013 vom BMBF gefördert und gemeinsam mit der HWR Berlin durchgeführt. In diesem Rahmen wurde an der Technischen Hochschule Wildau unter Leitung von Frau Prof. Dr. Margit Scholl ein elektronisches Lehrwerk in Form eines videobasierten Onlinekurses $\mathrm{zu}$ interkulturellen Kompetenzen für KMU und KKU in Brandenburg erarbeitet, das inhaltlich und methodisch vielfältigen Anforderungen an Weiterbildungsangeboten im Themenfeld Interkulturalität Rechnung tragen und dem Lehr- und Lernformat Blended Learning genügen sollte.

Vornehmlich um die Anforderungen der KKU/KMU in Brandenburg kennenzulernen, wurden im Teilprojekt „Kultur und Technik“ zwei Umfragen erstellt. Auf der Wirtschaftsmesse TH Connect an der TH Wildau ${ }^{4}$ wurden 2010/2011 Daten von 30 Brandenburger Unternehmen zum Thema Weiterbildungsmaßnahmen und Trainingsinhalte sowie Bedarfe im Bereich der interkulturellen Weiterbildung abgefragt. Die zweite Umfrage „Qualität und Kompetenz in interkulturellen Bildungsmaßnahmen" wurde im Sommer 2012 für interkulturelle Trainer $^{5}$ und in Weiterbildungsmaßnahmen professionell Tätige als Online-Umfrage durchgeführt. Frage- stellung war, welche interkulturellen Kenntnisse und Kompetenzen von den o.g. Experten als substantiell für Geschäftsleute in der internationalen Wirtschaft erachtet werden.

Die Thematik der interkulturellen Weiterbildung wurde somit von zwei Seiten beleuchtet, um Einschätzungen bzw. Einstellungen von Unternehmensmitarbeitern und von Weiterbildungsanbietern zur Situation und zu relevanten Inhalten und Methoden interkultureller Qualifikationen zu eruieren. Die Ergebnisse dienten der Vorbereitung eines onlinegestützten Lernangebotes zum Erwerb interkultureller Schlüsselkompetenzen für Mitarbeiter/-innen in Brandenburger KKU/KMU.

\section{Folgende Fragen waren hierbei von zentraler Bedeutung:}

1. Inwieweit ist den KKU/KMU in Brandenburg Interkulturalität als Faktor für den wirtschaftlichen Erfolg bewusst?

2. In welchem Umfang wird in KKU/ KMU in Brandenburg Weiterbildung bereits mit Methoden des Blended Learning durchgeführt?

3. Wie sollte ein attraktives Lernangebot aussehen, das mit geringem Zeitaufwand durch Trainer und Mitarbeiter in Unternehmen abgerufen werden kann?

In unserem Beitrag ${ }^{6}$ werden zunächst die Ergebnisse beider Umfragen dargestellt und daraus die wesentlichen Positionen für die Erarbeitung des anvisierten Onlinekurses abgeleitet. Darauf folgt eine kurze Beschreibung des Lehrmaterials. Anschließend werden die Möglichkeiten und Grenzen eines Onlinekurses im Rahmen von Blended Learning erörtert.

\section{ERGEBNISSE DER UMFRAGEN ${ }^{7}$}

\section{II.I DIE SICHT DER UNTERNEHMEN (ERSTE UMFRAGE)}

Die Umfrage in den KMU erfolgte mittels Fragebögen und Interviews. In der Fragebogenbefragung ging es zunächst darum zu erfahren, ob seitens der KMU ein Interesse an Weiterbildungen besteht, welche Arbeitsbereiche und Themen von Interesse sein könnten. Darüber hinaus sollte der Umfang von Weiterbildung durch Blended-Learning-Szenarien ermittelt werden. In Bezug auf Interkulturalität wurde erfragt, ob die Unternehmen ausländische Mitarbeiter beschäftigen, Geschäftskontakte ins Ausland pflegen und ob die unternehmensinterne Weiterbildung an die Internationalisierung angepasst wird. Anschließend wurden persönliche Gespräche mit prototypischen Gesprächspartnern der mittleren Leitungsebene geführt.

Durch den Fragebogen wurde deutlich, dass die Unternehmen an Weiterbildungen interessiert sind, aber bis dato nur ungenaue Vorstellungen von den Methoden des Blended Learnings hatten. Außerdem stellte sich heraus, dass der Terminus Blended Learning wenig geläufig war, der deswegen durch den Begriff E-Learning ersetzt wurde. Es wurde ermittelt, dass nur 20 Prozent (6 von 30) der Befragten E-Learning bei der Weiterbildung einsetzten.

Geschäftskontakte ins Ausland sind bei den KKU/KMU in Brandenburg nur in Einzelfällen vorhanden, ausländische Mitarbeiterinnen und Mitarbeiter wurden in den KKU/KMU Brandenburgs zwar beschäftigt ${ }^{8}$, doch wird Interkulturalität von den KMU bisher nicht als Weiterbildungsthema wahrgenommen. Alle Befragten verstanden sich zwar als kulturinteressiert und wollten ihre Mitarbeiter weiterbilden, allerdings haben Investitionen in das Be-

\footnotetext{
${ }^{3}$ FHProfUnt $=$ Forschung an Fachhochschulen mit Unternehmen. S. URL: http://www.bmbf.de/de/1952.php?hilite=FHProfUnt

${ }^{4} \mathrm{TH}$ Connect $2013=$ URL: http://thconnect.de/ausstellerservice/angebot /

${ }^{5}$ Unter dem Begriff „Trainer“ werden hier Personen subsumiert, die Weiterbildungsmaßnahmen in verschiedenen Formen anbieten, also Trainerinnen und Trainer, Coaches, Beraterinnen und Berater, Dozentinnen und Dozenten, Expertinnen und Experten auf dem Gebiet der Interkulturellen Kommunikation.

${ }^{6}$ Der komplette Abschlussbericht des Projekts InterKomp KMU 2.0 ist bereits veröffentlicht, s. Scholl (2013).

7 Die Umfragen werden hier leicht komprimiert dargestellt. Eine ausführlichere Beschreibung findet sich in der unveröffentlichten Projektdokumentation, die bei der Projektleiterin, Frau Prof. Dr. Margit Scholl, hinterlegt ist.

${ }^{8}$ In $54 \%$ der 30 befragten Unternehmen ist die Belegschaft zu höchstens $14 \%$ nicht-deutscher Herkunft, während in lediglich $10 \%$ der KMU die Belegschaft zu mindestens $30 \%$ nicht-deutscher Herkunft sind.
} 
triebsvermögen deutlichen Vorrang. Dennoch haben $60 \%$ der Befragten Weiterbildungsbedarf bestätigt.

Die Interviews zeigten, dass die Unternehmen zwar fachspezifisch aus- und weiterbilden, jedoch nicht in übergeordneten Themen wie Interkulturalität. Darüber hinaus unterscheiden sich die Weiterbildungsmaßnahmen je nach Unternehmen, selbst wenn sie zur gleichen Branche gehören. So wird bisher in Handwerksbetrieben sehr traditionell weitergebildet, durch Hands-On-Trainings und Kommunikation zwischen Meister und Gesellen. Es wurde deutlich, dass die Methoden und Inhalte eines zu erstellenden Kurses zum Thema Interkulturalität den unterschiedlichen weiterbildungsspezifischen Rahmenbedingungen der Unternehmen Rechnung tragen müssen, insbesondere in Bezug auf den Bildungsstand der Mitarbeiter, die Verfügbarkeit von Technik und die Bereitschaft der Unternehmensleitung, Mitarbeiter für Weiterbildungen freizustellen.

Auch wenn der Ausländeranteil in den Brandenburger Unternehmen bislang noch gering ${ }^{9}$ ist, stellten die Interviewten fest, dass die KKU/KMU in Brandenburg sich in naher Zukunft auf unterschiedliche Mentalitäten einstellen müssen. So wird z.B. wahrgenommen, dass aus Osteuropa ${ }^{10}$ stammenden Fachkräfte in den befragten Unternehmen in der Regel über gute Sprach- und Fachkompetenzen verfügen, aber in Bezug auf das Kommunikations- und Konfliktverhalten kulturspezifische Besonderheiten aufweisen, wodurch gravierende Missverständnisse im Arbeitsprozess entstehen können. Hierfür wären die Personalverantwortlichen zu sensibilisieren, z.B. im Rahmen einer interkulturellen Weiterbildung.

Das onlinebasierte Lernen spielt bisher eine untergeordnete Rolle, obwohl Bedarf besteht. Gerade marktspezifische onlinebasierte Weiterbildungen wären nach Meinung der Interviewpartner besonders sinnvoll. Die Interviewten waren sich beispielsweise bewusst, dass bei Geschäftskontakten z.B. nach China Kulturwissen erforderlich ist (z.B. Verhandlungstaktik und Rituale, Formen der Kontaktanbahnung u.a.). Neben interkulturellen Themen spielen aber auch die rechtlichen, insbesondere zollrechtlichen, steuerlichen und logistischen Bedingungen vor Ort eine Rolle.

Die Interviewten sahen die Defizite in ihren Unternehmen, indem sie diese mit den Praktiken von größeren Unternehmen verglichen, in denen interkulturelle Coaches Auslandsgeschäfte begleiten und für die interkulturelle Weiterbildung der Mitarbeiter mehr getan wird, z.B. auch durch die Beschaffung spezifischer Lernsoftware für das Selbststudium. KKU/KMU sind nach Aussage der Gesprächspartner vor allem im alltäglichen Geschäft tätig, wobei Zeitmangel und knappes Budget interkulturelle Fragen in den Hintergrund drängen.

\section{II.II DIE SICHT DER WEITERBILDUNGS- ANBIETER (ZWEITE UMFRAGE)}

Ziel der zweiten Befragung war, sich ein Bild von der Unterrichtspraxis der Personen zu machen, die interkulturelle Weiterbildungen professionell deutschlandweit durchführen. Sie folgte der Annahme, dass interkulturelle Trainer sich in ihrem Vorgehen, in Zielstellungen, Inhalten, Methoden und didaktischem Herangehen nach ihren Zielgruppen, nach den Bedarfen von Teilnehmenden bzw. von Auftraggebern richten und ihr Trainingskonzept $^{11}$ entsprechend gestalten, da der Lernerfolg bekanntlich mit dem Maß der Übereinstimmung von Erwartung der Trainingsteilnehmenden und der Trainingsgestaltung korreliert (vgl. Barmeyer 2000, Brislin 2000, Kinast 2009, Kováčová 2010).

Die Befragten arbeiten mehrheitlich in der Wirtschaft, 29 Befragte arbeiten in
Großunternehmen und 25 in KMU, davon zwei in Berlin/Brandenburg bzw. nur in Brandenburg. Weitere Befragte sind in Verwaltung und Hochschulen tätig. Branchenspezifisch wurden v. a. Automobilindustrie (50\%), IT-Branche (28\%), Luftfahrt (22\%), Finanzwesen (22\%), Medizin und Pharma (17\%) sowie zu geringeren Anteilen Chemie, Maschinenbau, Verwaltung, erneuerbare Energien u. a. angegeben (Mehrfachnennungen waren möglich).

Der Fragebogen ${ }^{12}$ wurde von 61 Personen beantwortet, allerdings in unterschiedlicher Ausführlichkeit ${ }^{13}$. Auf die Frage, welche Lehr- bzw. Lerninhalte die Befragten für zentral erachten, weisen $60 \%$ der Antworten (nur von 10 Personen beantwortet) auf die Bedeutung der emotiv-affektiven Seite des Kompetenzerwerbs und auf empathische Fähigkeiten hin, darunter Offenheit, Bewusstsein, Umgang mit Unsicherheit und weitere emotive Erfahrungswerte. $20 \%$ haben explizit die kognitive Wissenskomponente in den Vordergrund des Lernens gestellt. In den restlichen Äußerungen sind andere Aspekte genannt worden. Als wichtig für die kognitive Lernebene werden „relevantes Wissen“ - teils in Einzelbereichen wie Kommunikation und Konfliktverhalten oder das Wissen um ethnorelative Faktoren und auch kulturelles Hintergrundwissen, „um Verhalten einzuschätzen“ - angegeben. Auf der operativen Ebene werden als Lernziel überwiegend gute Kommunikationsfähigkeiten (Gesprächsführung, Zuhören) und adäquater Umgang in konkreten Situationen bzw. interkulturellen Interaktionen genannt.

Die Zielgruppe der Trainer wurde dazu befragt, ob es in ihrem Trainingserleben Situationen gab bzw. gibt, in denen Teilnehmende oder Auftraggeber andere Erwartungen an die interkulturellen Bildungsmaßnahmen hatten als die Trainer, sei es inhaltlich oder methodisch. Auf diese Frage antworteten lediglich 11 Personen, von ihnen nur eine Person mit „Nein“, 10 mit

\footnotetext{
${ }^{9}$ Zur Zeit der Befragung waren es 1,55\% (Statistisches Jahrbuch Brandenburg 2011, S. 90 ff).

${ }^{10}$ Die meisten ausländische Arbeitskräfte im Land Brandenburg kommen laut Statistisches Jahrbuch Brandenburg (2011) aus Osteuropa (Polen 1.946, Russen 630, Ukrainer 471), gefolgt von Menschen aus asiatischen Ländern (1.844) und der Türkei (1.402).

Bzw. ihre Begleitung, Beratung, Seminare sowie ggf. Konfliktanalyse und interkulturelle Mediation u.a.

${ }^{12}$ Die Konzeption und Auswertung der Umfrage wurde unter Mitarbeit von der im Projekt beschäftigten Studentin Franziska Koltermann durchgeführt

${ }^{13}$ Insgesamt haben 81 in Deutschland tätigen Personen den Fragebogen aufgerufen, 41 Personen haben mit der Beantwortung begonnen, 20 Befragte haben die zentralen Bereiche beantwortet.
} 
"Ja“ bzw. „Gelegentlich“; sie gaben als Begründung an, dass Trainingsteilnehmende häufig Patentrezepte bzw. Regeln oder ToDo's (6 Befragte) oder auch einen Sprachkurs erwarten (1) bzw. gar keine Vorstellung vom Kursinhalt haben (1).

$60 \%$ der befragten Trainer gaben an, dass sich Lehr- bzw. Lerninhalte in den Trainings wenig zielgruppen- und branchenspezifisch unterscheiden. $40 \%$ hoben Unterschiede in den Lerninhalten sowie Bedürfnissen und Rollen von Teilnehmern hervor und wiesen auf divergente Trainingsziele hin. Ihre Trainingsteilnehmenden sind zu knapp drei Vierteln Manager und Führungskräfte aus der Wirtschaft (Groß-, Mittel- und Kleinunternehmen), darunter auch Expatriates, weiter zu gut einem Drittel Firmenmitarbeiter (Fachkräfte, Sachbearbeiter) sowie zu wenigen Anteilen Flüchtlinge, Migranten, Missionare, Studierende u.a.

Etwa die Hälfte der Befragten nannte vorwiegend Gespräche und Diskussionen als Arbeitsmethoden, ein Drittel der Trainer wendet auch Rollenspiele und Szenarien als Praxis des interaktiven, erfahrungsorientierten Lernens an. Diese Angaben stehen interessanterweise im umgekehrten Verhältnis zu den Nennungen in Bezug auf Lerninhalte und angestrebte Kompetenzen, bei denen die affektive Lernebene als besonders bedeutsam bezeichnet wurde ${ }^{14}$. Der stärkere Einsatz von diskursiven Methoden könnte allerdings auch auf die Präferenzen der Teilnehmer zurückgeführt werden (vgl. Barmeyer 2000). Immerhin fünf von sechs Antworten im Bereich "Sozialform" weisen Gruppenarbeit oder Paararbeit als kooperative Lernformen aus.

Mediennutzung erfolgt sowohl in klassischer Art und Weise (Flipchart, Kärtchen, Figuren etc.) als auch über elektronische (Powerpoint) und audiovisuelle Medien.

In Bezug auf Lehr- und Lernformate gaben 13 Personen (von 13 Nennungen) an, ausschließlich Präsenztrainings und keine Online-Trainings durchzuführen, lediglich eine Person nennt zusätzlich „Online (Skype)“. Aufgrund dieser Angaben kann keine Auswertung zu möglichen Online-Trainingsszenarien erfolgen. Aus den Angaben der Befragten lässt sich jedoch schließen, dass das Medium Internet hauptsächlich als Informationsbeschaffungsmedium, Wissensspeicher und eher nicht als Interaktionsinstrument genutzt wird, d. h. Dokumente (Bilder, Filme) werden geladen, Informationen werden abgefragt, doch interaktive Formen wie Virtual Classroom, Online-Diskussionen, Webinar und weitere Online-Kommunikationsformen werden - so die Schlussfolgerung - nicht als Trainingselement genutzt und auch nicht positiv eingeschätzt. Zitate von Befragten zeugen davon: „Der Nachteil von Online-Maßnahmen ist, dass das emotionale Erleben fehlt.“ " ... kein Dialog ...“; „Online finde ich keine gute Möglichkeit zur interkulturellen Sensibilisierung, da es um innere Haltungen und Werte geht - jeder kann sich online besser verstecken." Die Wirkung sei diffus und nicht greifbar. Eine Person spricht sich gegen die Online-Trainings mit dem Argument „mehr Zeitaufwand ... für Qualität, Effektivität“ aus. Als Vorteile von Online-Training werden zumindest organisatorische Aspekte wie Kosten- und Zeitersparnis durch entfallende Anreise bzw. Ortsunabhängigkeit, Flexibilität und geringerer logistischer Aufwand angeführt.

\section{II.III SYNOPSE}

Zur ersten Umfrage: In KKU/KMU in Brandenburg stehen bisher Trainings bzw. Weiterbildungsmaßnahmen im Vordergrund, die ein momentanes Problem der Mitarbeiter beseitigen, z.B. Schulungen in handwerklichen Techniken oder in Softwareanwendungen für den täglichen Gebrauch. Hierbei ist von Bedeutung, auf dem aktuellen Stand zu sein und mit Mitarbeitern unterschiedlicher Kulturen erfolgreich agieren zu können. Das Budget für Weiterbildungen ist jedoch limitiert.

Die Wünsche zu interkulturellen Weiterbildungsmaßnahmen fokussierten auf Vermittlung von Informationen („fremde Rituale“) bzw. einer Art „Handwerk-Tools“ („Verhandlungstechnik"). Das Bewusstsein dafür, was Interkulturalität bedeutet und auf welchen Wegen eine reflektierte interkulturelle Kompetenz zu erwerben wäre, ist laut Selbsteinschätzung noch weit unterentwickelt. Die Interviewpartner sehen deshalb auch ihre Aufgabe darin, ein entsprechendes Verständnis bei $\mathrm{KKU} / \mathrm{KMU} \mathrm{zu}$ wecken und zu entwickeln. Einen einheitlichen, dezidierten Weiterbildungsbedarf an interkultureller Qualifizierung, auch innerhalb einzelner KKU/KMU-Branchen, konnte aus dem Datenmaterial nicht abgeleitet werden.

Ebenso hat sich gezeigt, dass die Befragten kaum klare Vorstellungen über Konzepte, Methoden und Möglichkeiten des Blended Learnings haben und dementsprechend auch keinen besonderen Weiterbildungsbedarf sahen. Allerdings haben sie eingeräumt, dass in den KMU sowohl gegenüber Blended-Learning-Konzepten als auch gegenüber interkulturellen Qualifizierungsmaßnahmen gewisse Vorbehalte bestehen, die im zweiten Falle sogar noch stärker ausgeprägt sind.

Zur zweiten Umfrage: Die Antworten der Trainer lassen eine Professionalität in ihrem Metier erkennen. Eine stärkere Gewichtung der emotiven Komponente des Kompetenzerwerbs während des Trainingsgeschehens entspricht der Realität des interkulturellen Lernens. Die Lernprozesse verlaufen in mehreren Phasen (s. Bolten, 2007:80ff, Thomas, 2014:166ff) und sind den unvorbereiteten Teilnehmern i.d.R. zunächst kaum bewusst (s. dazu Kap 5). Daraus resultiert das bekannte Missverständnis, dem Interkulturalisten in ihrer Dozenten- bzw. Trainertätigkeit immer wieder begegnen, nämlich die Erwartungen einiger Teilnehmer, dass man schnell "Tricks“ u.ä. für die Geschäfte mit kulturell Anderen erlernt. Dies bedeutet jedoch eher eine Utilitarisierung des Kulturwissens als interkulturelle Kompetenz (vgl. Rösch, 2013). Hiermit korrespondiert auch, dass es einige Trainer als besonders schwierig ansehen, in der Veranstaltung Hintergründe (Basiswis- 
sen) interkultureller Kompetenzen wie Werte und Haltungen zu vermitteln bzw. die Teilnehmenden für andere Kulturen überhaupt zu sensibilisieren. Die Aussagen der Trainer, dass die Lehr- und Lerninhalte nur wenig zielgruppen- und branchenspezifisch variieren, sprechen dafür, dass ein bestimmter Themenkanon und Methodenpool erforderlich ist, um das Lernziel - interkulturelle Kompetenz für die praktische Gestaltung der interkulturellen Zusammenarbeit - zu erreichen.

Fazit: Nach Auswertung beider Umfragen kann zunächst festgehalten werden, dass Vorstellungen sowie Wünsche und Erwartungen der Lernerseite mit Angeboten und Vorstellungen der Anbieterseite eher divergieren. Es liegt jedoch nahe, diese Situation als Kommunikationsdefizit zu interpretieren. Obwohl den Interessierten eine relativ große Auswahl an Bildungsmöglichkeiten in Bezug auf interkulturelle Thematik und E-Learning-Konzepte zur Verfügung steht, scheinen die diesbezüglichen Informationen in den KMU/ KKU nicht bekannt genug zu sein und die Bedenken aufgrund von Zeit- und Geldmangel derzeit noch zu überwiegen.

\section{LEITGEDANKEN BEI ERSTELLUNG DES ONLINEKURSES}

Das Gemeinschaftsvorhaben „InterKomp KMU 2.0" sollte helfen, den besonderen Rahmenbedingungen der KKU/KMU Rechnung zu tragen und die interkulturellen Kompetenzen ihrer Mitarbeiter zu verbessern. Gerade der bevorstehende demographische Wandel mit rückläufigen Studentenzahlen, der unterdessen mit einer verstärkten Multikulturalisierung der Gesellschaft einhergeht, wird die KKU/KMU in der Region vor große Herausforderungen stellen.

Die Entwicklung in Deutschland wird sicherlich auch vom Zuzug sowohl hochqualifizierter Experten als auch Studierender und Absolventen aus dem Ausland abhängig sein. Hiervon können die KKU/KMU profitieren, wenn es gelingt, eine neue Qualität an interkultureller Kompetenz kontextbezogen und handlungsorientiert zu etablieren. Ohne die Kompetenz, internationale
Fachkräfte zu integrieren, können KKU/ KMU nicht dauerhaft wettbewerbsfähig bleiben. Die Informationen über die Möglichkeit der interkulturellen Weiterbildung müssen an die KMU der Region in einer angemessenen Form weitergegeben werden.

Als Ergebnis der beschriebenen Umfragen wurden folgende Anforderungen an die Lehrmaterialien gestellt:

1. Geringe Kosten: Angesichts des Kostendrucks in den Brandenburger KMU sollte die Anschaffung von Lehrmaterialien nur geringe Kosten verursachen. Auch für die Trainer ist der Kostenfaktor entscheidend, da sie als Freiberufler kaum über finanzielle und zeitliche Möglichkeiten verfügen, für ihre Kunden webbasierte Lernkurse zu entwickeln.

\section{Kurze Lerneinheiten bei freier} Zeiteinteilung: Die Mitarbeiter in KMU stehen unter hohem Arbeitsdruck, der oftmals nur wenig Zeit für Weiterbildung lässt. Zeitaufwändige Sensibilisierungsmaßnahmen im Bereich Interkulturalität sind insofern problematisch. Daher sollten die Lerneinheiten möglichst kurz (5-15 Minuten) und jederzeit aufrufbar sein.

3. Einfache kognitive und organisatorische Zugänglichkeit: Die einfache Zugänglichkeit der Lerneinheiten ist ein wesentlicher Faktor für die Akzeptanz für Mitarbeiter und Trainer. Deshalb wurde die Erstellung von kurzen Lernvideos als die Möglichkeit gesehen, das Thema Interkulturalität audio-visuell und damit kognitiv ansprechend zu gestalten. Als technische Lösung bot sich ein Onlinekurs an, auf den über einen Internetzugang zugegriffen werden kann.

4. Individuelles Lerntempo und Modularisierung: Die Mitarbeiter (auch Personalabteilungen) und Trainer sollten sich die Inhalte/Themen aussuchen können, die sie selbst aktuell für relevant empfinden. Außerdem sollten die Lernenden ihr individuelles Lerntempo bestimmen können. Eine übersichtliche Strukturierung der Lerneinheiten sowie die Verfügbarkeit von einzelnen Lernvideos soll beides ermöglichen. Für Mitarbeiter und Trainer, die sich vertiefend mit einem Thema auseinandersetzen auseinandersetzen möchten, sollen zudem etwas längere Lerntexte bereitstehen. Diese wiederum sind für Trainer interessant, weil sie als Lernunterlagen im Training verwendet werden können.

\section{Beispiele aus dem konkreten Ar-} beitsumfeld: Für Mitarbeiter ist die konkrete und anschauliche Beschreibung einer Kulturdimension bzw. einer interkulturellen Interaktion und deren Analyse wichtig, um Lernerfolg zu erzielen. Trainer sollten in unserem Lehrmaterial didaktisch verwendbare Beispiele finden und in ihren Trainings für Unternehmen einsetzen können. Daher wurde bei der Erstellung der Lernvideos und der vertiefenden Lerntexte darauf geachtet, konkrete Beispiele auch audio-visuell darzustellen.

\section{Grundlage für Blended-Learning-} Szenarien schaffen: Blended Learning wird als Kombination von Lerneinheiten in Präsenzphasen und in E-Learning-Phasen gesehen. Jedoch wird diese Lernform weder in KMU noch bei Interkulturellen Trainern genutzt. In großen Unternehmen und in anderen Trainingsbranchen findet Blended Learning bereits breite Verwendung (z.B. zu Arbeitssicherheitsthemen). Ein onlinebasierter Kurs mit interkultureller Thematik würde die Grundlage für die Durchführung von Blended-Learning-Trainings schaffen, da einzelne Module auch vor oder nach einem Seminar bearbeitet werden könnten.

Im Ergebnis der geschilderten Anforderungen wurde ein videobasierter Onlinekurs entwickelt, der Mitarbeitern und Trainern frei zugänglich ist. Interessierte Nutzer sollen über die Projektwebseite auf der Homepage der TH Wildau dorthin gelangen können:

kmu-interkomp20.th-wildau.de/

?page_id=313

Nutzername: kmugast

Kennwort: kmuinterkomp20 
Das Projektteam der TH Wildau stellt eine weitreichende Verteilung dieses Onlinekurses sicher. Dafür werden Lizenzrechte der Creative Commons 3.0 genutzt, d.h. dass Änderungen am Werk möglich sind, wenn diese von uns freigegeben werden, und dass die Inhalte des Kurses auch kommerziell genutzt werden können.

Dabei ist zu beachten, dass die Onlinekurse interkulturelle Präsenztrainings nicht ersetzen können. Vielmehr wird es für individuelle Fragestellungen von KMU immer notwendig bleiben, spezialisierte Trainer zu engagieren. In diesem Sinne sehen wir unsere Onlinekurse als einen wichtigen Schritt zur Bereicherung und Ergänzung vorhandener Methoden und Lernformate.

\section{DER ONLINEKURS INTERKOMP KMU 2.0 - INTERKULTURELLES LERNEN UND ARBEITEN}

Der Onlinekurs „Interkulturelles Lernen und Arbeiten" besteht aus Teil 1 „Einführung in die Interkulturelle Kommunikation“ und Teil 2 "Interkulturelles Arbeiten“. Er umfasst 12 Lernvideos auf der Lernplattform der TH Wildau sowie auf YouTube, enthält Aufgaben zur Reflexion und Anwendung sowie ca. 120 Seiten vertiefendes Lernmaterial ${ }^{15}$.

Ausgangspunkt der Lerneinheiten waren Texte zu Grundbegriffen, Problemen und Fragestellungen der interkulturellen Kommunikation. Es ging dabei um die Aspekte der Kultur, die in den interkulturellen Interaktionen für Missverständnisse und Misserfolge verantwortlich sein können und deshalb besondere Aufmerksamkeit verdienen. Es sind Themen wie z.B. Kulturverständnis, Stereotype, kulturbedingte Kommunikationsstile, Umgang mit Macht, Zeit, Regeln und Strukturen sowie zwischenmenschliche Beziehungen in der interkulturellen Zusammenarbeit. Diese thematischen Schwerpunkte, die den zusammenhängenden Themenkanon des interkulturellen Lernens (s.o.) gleichsam abbilden, wurden in acht Hauptthemen gegliedert und in 12 Module übertragen.
Die Lernvideos sind mit 5 bis 15 Minuten kurz gestaltet und vermitteln in ansprechender Form relevantes Wissen zum Grundverständnis des interkulturellen Lernens. Die zugrunde liegenden Texte wurden zunächst in das Präsentationsformat PowerPoint umgewandelt, um diese anschließend zu vertonen. Die entstandenen Lernvideos sind zudem durch einige Links angereichert und enthalten u.a. Rollenspiel-Videos mit anschaulichen Beispielen. Flankiert werden die Lernvideos außerdem mit vertiefenden Texten. In den Vertiefungstexten finden sich viele anschauliche Beispiele, die den Nutzern das jeweilige Thema detailliert vermitteln. Die Reflexionsfragen sind knapp formuliert und dienen der nachträglichen Verarbeitung des Gelernten. Die Anordnung „Lernvideos“, "Reflexion“, „Vertiefung“ bietet verschiedene Ebenen der kognitiven Elaboration und soll eine übersichtliche Navigation gewähren.

Die Lernvideos bieten zudem ein Inhaltsverzeichnis und werden durch Aufgabenstellungen und Forendiskussionen unterstützt. Um auch den Zugriff über Smartphone zu gewährleisten, können die Lernvideos auf YouTube hochgeladen und dann im Lernkurs verlinkt werden, so dass sie auch auf kleinen Displays betrachtet werden können.

Insgesamt sollen durch die Mischung aus Lernvideos, Reflexionsfragen und Vertiefungstexten unterschiedliche Lerntypen und Lernbedürfnisse angesprochen werden. Die Erklärungen sollen trotz ihrer Komplexität verständlich bleiben, auch für KMUMitarbeiter, die sich bisher weder mit webbasiertem Lernen noch mit interkulturellen Themen auseinander gesetzt haben.

Der Kurs wurde im Laufe der Arbeit in einer Beta-Version getestet und auch von mehreren Seiten evaluiert. Das Feedback floss in die weitere Entwicklung des Onlinekurses ein. Der veröffentlichte Kurs wurde von den Nutzern insgesamt positiv aufgenommen (ausführlicher dazu s. Scholl, 2013:69-73).

\section{E-LEARNING ALS EINE MÖGLICHKEIT DER INTERKULTURELLEN KOMPE- TENZVERMITTLUNG}

Der Einsatz von E-Learning in der interkulturellen Bildung führt erst dann zum gewünschten Erfolg, wenn die Onlinekurse entsprechend dem Lernziel eingesetzt bzw. in die Vielfalt der Lernmethoden adäquat eingeordnet werden und die Kursteilnehmenden mit dem Prozess des interkulturellen Lernens vertraut sind.

\section{V.I ZUM EINSATZ IM BERUFSLEBEN}

Die interkulturellen Trainings sind bekanntlich ein Bestandteil des interkulturellen Lernens, aber sie gehören zu den unentbehrlichen und besonders häufig praktizierten Formen der interkulturellen Qualifizierung. Hinsichtlich des Einsatzortes u. -zwecks werden i.d.R. zwei Typen von Trainings unterschieden: 1. Trainings „off the job“ im Sinne von Weiterbildungsmaßnahmen, die keine konkreten Problemsituationen im Fokus haben, sondern grundsätzlich der kulturellen Kompetenzverbesserung dienen, und 2 . Trainings „on the job“, die bei Problemen vor Ort am Arbeitsplatz eingesetzt werden. Die beiden Trainingstypen werden als „Tools interkultureller Kompetenzverbesserung “ gesehen (Bolten, 2007:223):

Selbst wenn E-Learning in der Graphik nur unter "on the job“-Typ explizit erwähnt ist, kann aus der Beschreibung des von uns entwickelten Onlinekurses eindeutig abgeleitet werden, dass er für beide Trainingstypen gut geeignet ist. In beiden Fällen muss das Lernen von professionellen Trainern methodisch-didaktisch beratend begleitet werden, um an Lernbedarf, Lernkultur und Lernkontext angepasst zu werden.

\section{V.II ZUM LERNPROZESS}

Im Abschnitt 2.3 wurde die Frage nach den Erwartungen der Teilnehmer an interkulturelle Trainings und die damit verbundenen Missverständnisse in Bezug auf deren Inhalte und Methoden bereits angesprochen und auf die Lernphasen des interkulturellen 


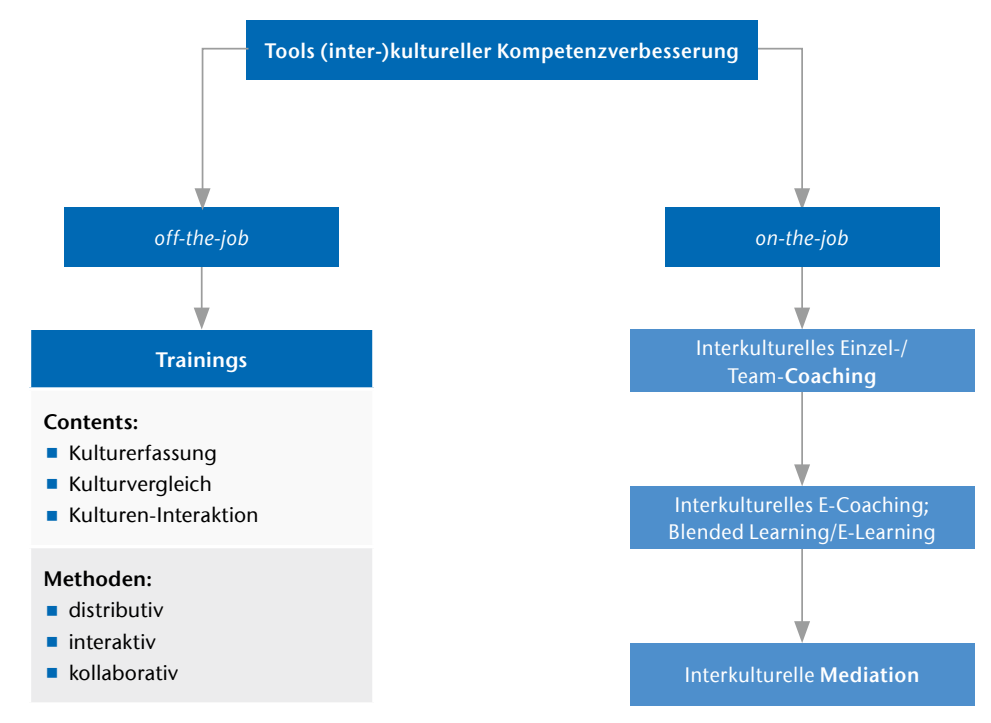

Abb. 1) Tools zur Verbesserung der (inter-)kulturellen Kompetenz (aus Bolten 2007:223)

Lernens hingewiesen. Darauf soll hier noch einmal eingegangen werden.

Der Wunsch vieler Trainingsteilnehmenden, die sich auf eine Zusammenarbeit mit kulturell Fremdem einstellen, besteht erfahrungsgemäß oft darin, die interkulturelle Kompetenz zeitsparend durch einige Informationen über eine fremde Kultur und durch „Tipps und Tricks“ als eine Art „Handwerk" zu erwerben. Das ist nicht überraschend und zeugt keineswegs von mangelnder Tiefgründigkeit. Denn dahinter steht ein verständliches Bedürfnis, durch Information und Wissen mehr Sicherheit im Umgang mit dem Fremden bzw. in der Fremde zu erlangen. Zu dieser Sicherheit kann man jedoch erst über den etwas längeren Weg der Auseinandersetzung mit der eigenen Kultur und der Weiterentwicklung von Fähigkeiten zur Selbstreflexion gelangen. Das ist den meisten Lernern nicht wirklich bewusst.

Der Prozess des interkulturellen Lernens ist in der Fachliteratur bereits aus unterschiedlichen Blickwinkeln hinlänglich beschrieben worden (vgl. dazu eine Übersicht bei Thomas, 2014: 166ff). Zur Anschauung wird das gut bekannte Phasenmodell von Leenen \& Grosch (1998: 40) bemüht, welches v.a. die Prozesshaftigkeit des Lernens im Fokus hat und leicht verständlich ist. Ordnet man die o.g. Wünsche seinen Zielebenen zu, so korrespondieren die Äußerungen der Lernenden in der bene als ihre Existenzform. Diese Ebene ist nicht unbedingt per Mausklick zugänglich; sie ist außerdem interpretationsbedürftig und interpretationsanfällig. Für die Entwicklung einer interkulturellen Handlungskompetenz für das Berufsleben im Rahmen der gegebenen Möglichkeiten bietet sich ein erfahrungsorientiertes Lehr-LernKonzept an mit Übungen, Fallstudien, Rollenspielen, Reflexion, Diskussion sowie Wissensvermittlung und Lehrgesprächen zwecks Anbindung an theoretische Inhalte. Es geht hierbei um Folgen von konkreten interkulturellen Erfahrungen der Lernenden, um Reflexion dieser Erfahrungen, um Heranziehen von Kulturwissen und Interpretationssystemen und schließlich um Ausarbeitung von kulturisomorphen bzw. kulturadäquaten Handlungsstrategien.

Tendenz mit der Phase 6 „Erweiterung der Handlungsoptionen“ "bzw. 7 "Interkulturelle Zusammenarbeit gestalten“:

1. Kulturgebundenheit erkennen

2. Fremdheit wahrnehmen

3. Das Eigene bewusst machen

4. Das Fremde adäquat deuten lernen

5. Verständnis für das Fremde entwickeln

6. Erweiterung der Handlungsoptionen

7. Interkulturelle Zusammenarbeit gestalten.

Die Zeit und der Diskussionsraum, die für die vorausgehenden Phasen $1-5$ objektiv benötigt werden, sollen mit bedacht werden, denn die Materie Kultur im Sinne von Kommunikation hat zu einem großen Anteil die Gefühlse-
Aus der Graphik wird ersichtlich, dass sowohl kognitive als auch affektive Wege des Lernens erforderlich sind, um Lernfortschritte „erfahrbar“ zu machen. Die aufeinander aufbauenden Entwicklungsphasen geben insofern die Realität des Lernprozesses wieder. Es geht hierbei um die interkulturellen Erfahrungen der Lernenden (Auslandsaufenthalt, Simulationsspiel), um Reflexion dieser Erfahrungen (Diskussion, Lehrgespräch), um Heranziehen von Kulturwissen und Interpretationssystemen (Theorievermittlung) und schließlich um kulturadäquates Handeln (Erarbeitung von Handlungsoptionen in einer Arbeitsgruppe). Dieser Ansatz hat sich im Laufe der Jahre bewährt und stellt gewissermaßen ein

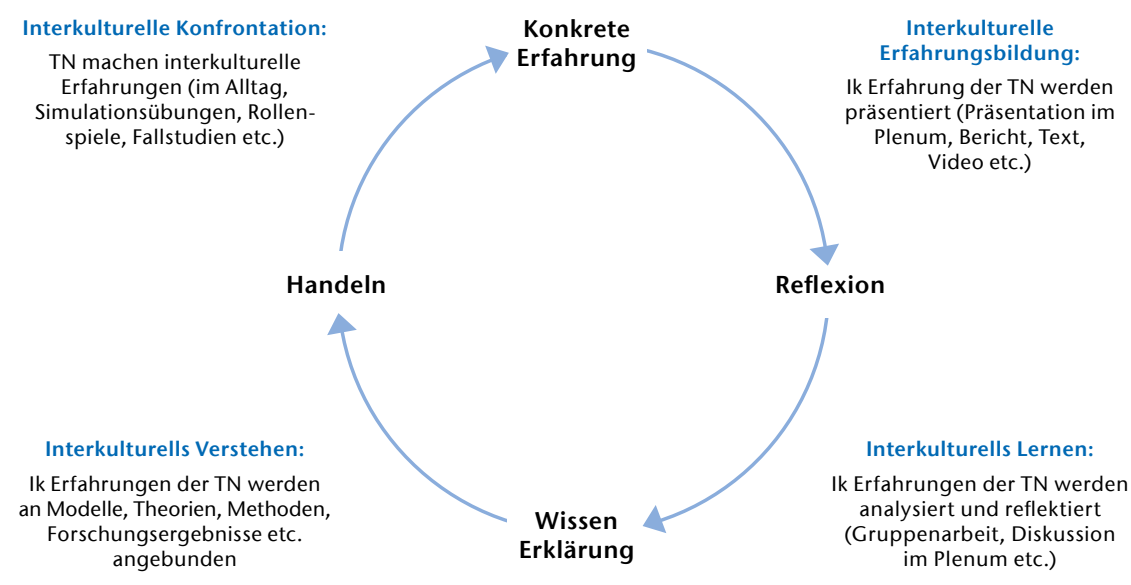

Abb. 2) Didaktisches Grundkonzept erfahrungsorientierten Lernens (aus Thomas \& Hößler 2007:86) 
Ergebnis der langjährigen Lehr-LernForschung sowie Lehrerfahrung dar (Thomas \& Hößler, 2007).

Der Bedarf an Diskussionen ist nicht nur aus der eigenen Lehrtätigkeit hinreichend bekannt, er wird sowohl von den befragten Trainern bestätigt (s.o. "Gespräch als Arbeitsmethode") als auch von den Nutzern (darunter auch Studenten, die aus eigenem Interesse den Onlinekurs studiert haben) gemeldet. Hier sei eine Feedback-Äußerung aus einem KMU zitiert:

"Jetzt kommt bei mir die Frage, wie ich z.B. die Kasachen dazu bringe, innerhalb des Termins doch noch zu den eigentlichen Arbeitspunkten zu kommen, ehe ich in den Flieger steige. Bei den meisten Lernstufen, wo es um das Verständnis für die andere Kultur geht, ist das Thema der Einbeziehung in den Projektverlauf als wichtig angekommen. Die Sensibilisierung für andere Kulturen und Abläufe ist da. Erkenntnis: Von da geht die Arbeit aber erst richtig los, wenn das Unternehmen ein interkulturelles Projekt durchführen möchte".

Hier wäre eine Diskussion mit den Seminarteilnehmern über diverse Handlungsoptionen bzw. die Gestaltung der Zusammenarbeit offensichtlich zielführend.

\section{V.III ZUM E-LEARNINC}

Im Bereich der interkulturellen Kompetenzvermittlung hat das E-Learning eine Entwicklung erfahren, die bis zu einem gewissen Grade mit der Entwicklung des Internets einhergeht. Je nach Verwendung der netzbasierten Medien werden drei Nutzungsszenarien unterschieden: E-Learning 1 . by distributing, 2 . by interacting und 3 . by collaboration. Idealtypisch kommen in der ersten Variante vorwiegend die Medien wie Präsentationen, Skripte, semi-interaktive Lernmodule u.a. zum Einsatz, in der zweiten sind es Lern-/Content-Managersysteme, interaktive Simulationen und Lernplattformen und in der dritten Virtual Classroom, Blogs, Wikis, Community Software u.a. (vgl. Bolten, 2010:402). In allen drei Szenarien wird die Rolle des gesteuerten Lernprozesses, in dem die Beziehung "Lehrende-Lernende“ in jeder Entwicklungsphase anders gestaltet werden kann, explizit hervorgehoben (vgl. ebd.). Außerdem wird festgestellt, dass die ersten zwei Szenarien, im Unterschied zu ELearning by collaboration, auch nicht zu "grundsätzlichen Neuentwicklungen von Aufgaben- und Übungstypen geführt haben“ (ebd. S. 403). Es gibt zwar Unterschiede im „Hinblick auf technologische Grundbedingungen“, jedoch sind die drei Entwicklungsphasen des E-Learning „nicht als qualitative Stufenfolge zu verstehen“. Sie koexistieren nebeneinander und werden je nach Nutzergruppe „in sehr unterschiedlicher Weise als mehr oder minder bedarfsgerecht eingeschätzt “ (ebd.).

Unser Onlinekurs steht eher in der Konzeption des E-Learning by distributing mit einigen Elementen des E-Learning by interacting und kann im Rahmen des Blended Learning am günstigsten durch eine didaktischmethodische Anpassung seitens des professionellen Trainers an die jeweilige Zielgruppe zum Einsatz kommen. Mit der Wahl der Konzeption des Onlinekurses tragen wir den Möglichkeiten der Brandenburger KKU/KMU in Bezug auf Zeit, Kosten, vorherige Erfahrungen mit E-Learning, technologische Grundbedingungen usw. im vollen Umfang Rechnung.

\section{ZUSAMMENFASSUNG}

Die Module unseres Onlinekurses lassen sich im Rahmen von Blended Learning in jeder Lernphase und in beiden o.g. Trainingstypen (off the job und on the job) mit Gewinn für die Lernenden einsetzen. Beim Erwerb von Kulturwissen und der Aneignung von Interpretationssystemen ist das besonders für das individuelle Lernen hilfreich. Ein webbasierter Onlinekurs stellt somit ein geeignetes „Instrument interkultureller Kompetenzverbesserung “ dar (vgl. Bolten, 2007:228f, 2010).

Um Missverständnissen vorzubeugen und eventuelle Enttäuschungen zu vermeiden, muss den Nutzern deutlich gemacht werden, dass ein solcher Onlinekurs nicht anstelle, sondern nur in Kombination mit Präsenzphasen bzw. Betreuung zum wirklichen Lernerfolg führt. Das diskursive Lernen, begleitet vom reflektierten Nachdenken bzw. vom kritischen Hinterfragen der Selbstverständlichkeiten sowie von der Besprechung einer kulturbedingten Konfliktsituation, ist ein fester Bestandteil des interkulturellen Lernprozesses. Erfahrungsgemäß können oft erst im Laufe der Diskussionen zielgruppenspezifische und lernkontextabhängige Lernschritte erreicht sowie kulturadäquate, problemlösungsorientierte Strategien für interkulturelles Handeln erarbeitet werden.

Insgesamt ist das Projektteam der TH Wildau nach etlichen Tests und Evaluationen des Onlinekurses sicher, dass "Interkulturelles Lernen und Arbeiten" Unternehmen und ihre Mitarbeiter sowie Trainer in Inhalten, Methoden und im webbasierten Lernen erfolgreich in unterschiedlichen Fort- und Weiterbildungsaktivitäten unterstützen kann. Die Notwendigkeit eines BlendedLearning-Szenarios, d.h. der Onlinekurs begleitet und ergänzt durch Trainings bzw. Beratungen, sollte jedoch den interessierten Nutzern unbedingt kommuniziert werden, um eine Nachhaltigkeit des Lernerfolgs zu sichern.

\section{LITERATUR}

Barmeyer, Christoph (2000): Interkulturelles Management und Lernstile. Campus, Frankfurt/M.

Bergemann, Niels/Sourisseaux, Andreas L. J. (Hrsg.) (2003): Interkulturelles Management. 3. Auflage. Springer, Berlin

Bolten, Jürgen (2007): Einführung in die Wirtschaftskommunikation. UTB, Basel u. a.

Bolten, Jürgen (2010): E-Learning, in: Weidemann, Arne/ Straub, Jürgen/ Notnagel, Steffi (Hrsg.) (2010): Wie lehrt man interkulturelle Kompetenz? Theorien, Methoden und Praxis in der Hochschulausbildung. Ein Handbuch. transcript, Bielefeld, S. $397-416$.

Brislin, Richard (2000): Understanding Culture's Influence on Behavior. 2nd ed. Fort Worth: Harcort.

Busch, Dominic (2013): Im Dispositiv interkultureller Kommunikation. Dilemmata und Perspektiven eines interdisziplinären Forschungsfeldes, transcript, Bielefeld.

Eß, Oliver (Hrsg.) (2010): Das Andere lehren - Handbuch Interkulturelle Handlungskompetenz. Waxmann, München.

Grünhage-Monetti, Matilde (Hrsg.) (2005): Interkulturelle Kompetenz in der Zuwanderungsgesellschaft: Fortbildungskonzepte für kommunale Verwaltungen und Migrantenorganisationen. Perspektive Praxis. Bertelsmann, Bielefeld.

Heringer, Jürgen (2007): Interkulturelle Kommunikation. Grundlagen und Konzepte, 2. Auflage, A. Francke Tübingen und Basel. 
Kinast, Eva (2009): Evaluation interkultureller Trainings. In: Thomas, Alexander /Kinast, Eva-Ulrike /SchrollMachl, Sylvia 2009 (Hrsg): Handbuch interkulturelle Kommunikation und Kooperation, Band 1: Grundlagen und Praxisfelder. S. 204-216. Vandenhoeck \& Ruprecht, Göttingen.

Koch, Eckart (2012): Interkulturelles Management. Fü Führungspraxis, Projektarbeit und Kommunikation. UVK, Utb, Konstanz/München:

Kováčová, Michaela (2010): Komparative Evaluation kulturspezifischer didaktischer und erfahrungsorientierte interkultureller Trainings. Lang, Frankfurt/Main. URL [12.08.2012] http://books.google.de/books?hl=de\&lr= \&id $=x F 512$ tw $558 C \& o i=$ fnd \&pg $=P A 11 \& d q=$ evaluation + interkultureller+Bildungsma [12.08.2012]

Kutschker, Michael/ Schmid, Stefan (2010): Internationales Management. 7. Auflage, Oldenbourg, München.

Leenen, W. R. \& Grosch, H. (1998): Bausteine zur Grund legung interkulturellen Lernens. In: Interkulturelles Lernen. Arbeitshilfen für die politische Bildung, S. 29-46. Bonn: Bundeszentrale für Politische Bildung.

Lüsebrink, Hans-Jürgen (2008): Interkulturelle Kommunikation - Interaktion, Fremdwahrnehmung, Kulturtransfer. 2. Auflage, J.B. Metzler, Stuttgart/Weimar.

Moosmüller, Alois (Hrsg.) (2007): Interkulturelle Kom munikation - Konturen einer wissenschaftlichen Diszip lin, Münchener Beiträge zur Interkulturellen Kommunikation, Band 20, Waxmann, Münster.

Müller, Stefan/ Gelbrich, Katja: Interkulturelle Kommunikation (2013): Weltbilder, Normen, Symbole, Rituale un Tabus. Vahlens, Reihe Handbücher der Wirtschafts- und Sozialwissenschaften, München

Otten, Matthias/Scheitza, Alexander/Cnyrim, Andre (Hrsg.) (2007): Interkulturelle Kompetenz im Wandel, Band 1+2, Frankfurt am Main/London.

Rösch, Olga (2013): Fragen der Vermittlung von Kulturwissen in der Ingenieurausbildung, in: Stumpf, S. Schuch, E./ Meyer, U. (Hrsg.), Technik und Kultur - Anwendungsorientierte Beiträge zu einem Spannungsfeld, Pabst Science Publichers, Lengerich, S. 149-166.

Rösch, Olga (Hrsg.) (2008): Technik und Kultur. Wildauer Schriftenreihe Interkulturelle Kommunikation, Band 6 , Berlin.

Roth, Julianne/ Köck Christoph (Hrsg.) (2004): Culture Communication Skills - Interkulturelle Kompetenz. Handbuch für Erwachsenenbildung. Bayerischer Volkshochschulverband e.V. München.

Rothlauf, Jürgen (2009): Interkulturelles Management. 3. Auflage. Oldenbourg, München

Scholl, Margit (Hrsg.) (2013): Konzipierung von modularen Weiterbildungsangeboten für KMU an der Schnittstelle von Technik und Kultur zum Thema Internationales IT-gestütztes Projekt- und Wissenschaftsmanagement im multikulturellen Umfeld. Shaker, Aachen.

Statistisches Jahrbuch Brandenburg (2011): Seite $90 \mathrm{ff}$ Kapitel 02: Erwerbstätigkeit. abrufbar unter www.statistik-berlin-brandenburg.de/Produkte/Jahrbuch/jb2011/ JB_201102_BB.pdf

Straub, Jürgen/ Weidemann, Arne/ Weidemann, Doris (Hrsg.) (2007): Handbuch Interkulturelle Kommunikation und Kompetenz: Grundbegriffe - Theorien - Anwendungsfelder. J.B. Metzler, Stuttgart/Weimar.

TH Connect $2013=$ URL: http://thconnect.de/ausstellerservice/angebot [12.08.2012]

Thomas, Alexander (2014): Wie Fremdes vertraut werden kann. Mit internationalen Geschäftspartnern zusammenarbeiten, Springer Gabler, Wiesbaden
Thomas, Alexander /Hößler, Ulrich (2007): Interkulturelle Qualifizierung an den Regensburger Hochschulen: Das Zusatzstudium Internationale Handlungskompetenz. Interculture Journal, 6 (3), 73-95. Verfügbar unter: http://www.interculture-journal.com/download/issues/2007_03.pdf [10.01.2014]

Thomas, Alexander at. al. (1995-2014): Trainingsreihe für Manager. Reihe Handlungskompetenz im Ausland. Göttingen: Vandenhoeck \& Ruprecht. (bisher 37 Bände erschienen).

Thomas, Alexander/ Kinast, Eva-Ulrike/ Schroll-Machl, Sylvia (Hrsg.) (2009): Handbuch interkulturelle Kommunikation und Kooperation, Bd.1 +2, 2. Auflage, Vandenhoeck \& Ruprecht, Göttingen.

Weidemann, Arne/ Straub, Jürgen/ Notnagel, Steff (Hrsg.) (2010): Wie lehrt man interkulturelle Kompetenz? Theorien, Methoden und Praxis in der Hochschulausbildung. Ein Handbuch. transcript, Bielefeld.

\section{AUTOREN}

Prof. Dr. phil. Olga Rösch

Fachgebiet Interkulturelle Kommunikation Fachbereich Ingenieur- und Naturwissenschaften (INW) www.tfh-wildau.de/roesch olga.roesch@th-wildau.de

\section{Prof. Dr. rer. nat. Margit Scholl}

Fachgebiet Wirtschafts- und Verwaltungsinformatik Fachbereich Wirtschaft, Informatik und Recht www.th-wildau.de/scholl margit.scholl@th-wildau.de

\section{Dr. phil. Bettina Strewe}

Freiberufliche Trainerin

InterKult Ost

www.bettina.strewe.de

bettina@strewe.de

\section{Christian Niemczik}

Wissenschaftlicher Mitarbeiter in Projekten

COMBLE, learn@wildau und InterKomp KMU 2.0

Fachbereich Wirtschaft, Informatik und Recht

christian.niemczik@th-wildau.de

\section{Anja Teske MA}

Wissenschaftliche Mitarbeiterin in Projekten InterKomp KMU 2.0 und iBaMs

Fachbereich Wirtschaft, Informatik und Recht ateske@th-wildau.de 